\title{
METODOLOGIA PROBLEMATIZADORA NA EDUCAÇÃO SEXUAL DE ADOLESCENTES NO SUL DO PARÁ
}

\section{ARTIGO ORIGINAL}

SILVA, Fernanda Alves da ${ }^{1}$, SOUSA, Ana Katryne Lopes $\mathrm{de}^{2}$, COSTA, Elem Cristina Silva da ${ }^{3}$, TAVARES, Sandra dos Santos ${ }^{4}$

SILVA, Fernanda Alves da. Et al. Metodologia problematizadora na educação sexual de adolescentes no sul do Pará. Revista Científica Multidisciplinar Núcleo do Conhecimento. Ano. 06, Ed. 12, Vol. 12, pp. 172-185. Dezembro de 2021. ISSN: 2448-0959, Link de acesso: https://www.nucleodoconhecimento.com.br/saude/educacao-sexual, $\quad$ DOI: 10.32749/nucleodoconhecimento.com.br/saude/educacao-sexual

\section{RESUMO}

O déficit no conhecimento sobre planejamento familiar e infecções sexualmente transmissíveis geram comportamentos de risco e tornam o público adolescente vulnerável à agravos em saúde. Nesse sentido, a enfermagem, em seu contexto de cuidado, atende as demandas e necessidades de cada grupo, projetando-se principalmente na promoção da saúde e prevenção de agravos. O presente estudo objetiva relatar as vivências de discentes de enfermagem na execução da Atividade Integradora do Curso de Enfermagem da Universidade do Estado do Pará (UEPA), no que tange a saúde sexual e reprodutiva do adolescente. Trata-se da descrição de uma ação de educação em saúde com adolescentes, utilizando a Metodologia Problematizadora Arco de Charles Maguerez, como método norteador, com as etapas: observação da realidade, levantamento dos pontos-chave, teorização, hipóteses de solução e intervenção/aplicação na realidade. Para guiar a execução

\footnotetext{
${ }^{1}$ Acadêmica de Enfermagem.

${ }^{2}$ Acadêmica de Enfermagem.

${ }^{3}$ Acadêmica de Enfermagem.

${ }^{4}$ Especialista em Docência Universitária com Ênfase em Saúde - UEPA. Graduada em Enfermagem pelo Centro Universitário de Anápolis - UniEvangélica.
} 
das etapas utilizou-se a seguinte pergunta: qual problema de saúde, no público adolescente, é mais evidente na realidade escolar e como a enfermagem pode colaborar para mudar esta realidade? Mediante a identificação e estudo do problema, desenvolveu-se uma ação de educação em saúde sobre sexualidade e gravidez na adolescência, com adolescentes a partir de 12 anos de idade, em uma escola de Ensino Fundamental II do sul do Pará. O déficit de conhecimento acerca das ISTS/AIDS e gravidez foram problemáticas observadas não só no relato dos profissionais da escola, mas no contato direto com os adolescentes durante a realização da ação educativa, em vista de não estarem familiarizados com as nomenclaturas das mesmas e a importância do preservativo de barreira como método preventivo. Ante o observado, vê-se a necessidade de ações que intensifiquem o cuidado com este público para a promoção da saúde e prevenção de agravos. Diante disso, o enfermeiro assume um papel de extrema importância como educador, colaborando para o desenvolvimento do autocuidado deste público. Nesse sentido, a ação desenvolvida colaborou para o conhecimento reflexivo e conscientização que podem propiciar condutas mais responsáveis, cooperando para a prevenção de gravidez na adolescência e ISTs/AIDS.

Palavras-chave: Educação em Saúde, Infecções Sexualmente Transmissíveis, Planejamento Familiar, Adolescente, Enfermeiro.

\section{INTRODUÇÃO}

A adolescência é uma etapa marcada por mudanças biopsicossociais, onde os relacionamentos e assuntos ligados à sexualidade ganham maior importância. Nesta fase, o adolescente corre maior risco de vivenciar práticas sexuais não seguras e de forma pouco responsável devido à falta de informação sobre o assunto, pela ausência de comunicação com familiares, pela existência de tabus e por receio de assumir uma vida sexual ante a família (GENZ et al., 2017).

Nota-se que, na adolescência, os indivíduos apresentam ansiedade em realizar atividades precocemente, majoritariamente sem preparação prévia. Essa ambição de viver intensamente pode implicar na iniciação sexual precoce, aumentando a 
vulnerabilidade deste grupo frente às Infecções Sexualmente Transmissíveis (ISTs) e AIDS e gravidez na adolescência, dentre outros riscos, que podem afetar sua vida futura (CARNEIRO et al., 2015).

Dados da Pesquisa de Indicadores Sociais das Mulheres do Brasil realizada pelo Instituto Brasileiro de Geografia e Estatística (IBGE) mostraram que a taxa de gravidez na adolescência ficou em 59 nascimentos a cada 1.000 mulheres de 15 a 19 anos de idade, em 2019. Na região Norte do país essa taxa passou de 84 , a maior segundo o estudo (IBGE, 2021).

De acordo com Lima; Moreira (2018), somente o controle e prevenção de agravos não são eficazes para diminuir situações de risco à população, mas sim a promoção da saúde, enfatizando, que durante a infância e adolescência, por ser um período de mudanças constantes no desenvolvimento do indivíduo, deveria haver uma atenção à saúde integrada. Nesse sentido, o profissional enfermeiro é uma figura indispensável para a formação crítico-reflexiva do cidadão.

A priori, ao ingressar em uma universidade, os discentes de enfermagem têm a perspectiva de uma atuação em hospitais, clínicas e Estratégias de Saúde da Família. Contudo, ao longo do curso, através de sua grade curricular e com a orientação e supervisão de docentes, esse paradigma é quebrado, trazendo à tona uma enfermagem com atuação ampla e em vários ambientes, que visa atender as demandas e necessidades de cada grupo, como o juvenil, projetando-se principalmente na promoção de saúde e prevenção de agravos (FERNANDES et al., 2019).

Nesse sentido, o objetivo deste trabalho é relatar as vivências de discentes de enfermagem na execução da Atividade Integradora do Curso de Enfermagem da Universidade do Estado do Pará (UEPA), no que tange a saúde sexual e reprodutiva do adolescente, em uma escola de Ensino Fundamental II no sul do Pará. 


\section{A ATIVIDADE INTEGRADORA DO CURSO DE ENFERMAGEM E A METODOLOGIA PROBLEMATIZADORA ARCO DE CHARLES MAGUEREZ}

O presente trabalho é um relato de experiência de acadêmicas de enfermagem na execução da Atividade Integradora do Curso de Enfermagem da Universidade do Estado do Pará (UEPA), na realização das etapas do Arco de Charles Maguerez, desde o problema observado na comunidade até a intervenção para a sua minimização. Por tratar-se de um trabalho do tipo relato de experiência, o presente não necessitou de apreciação do Comitê de Ética e Pesquisa.

A Metodologia Problematizadora Arco de Charles Maguerez compõe a base pedagógica e metodológica da Atividade Integradora proposta pelo Projeto Pedagógico do curso de Graduação em Enfermagem da UEPA. Esta estratégia é uma tendência pedagógica que norteia a formação do profissional da saúde com o objetivo de torná-lo um ser crítico-reflexivo, capaz de transformar sua realidade social por meio da ação-reflexão-ação (UEPA, 2013).

A Metodologia Problematizadora Arco de Charles Maguerez é composta por etapas que compreendem a observação da realidade, o levantamento dos pontos-chave, a teorização, as hipóteses de solução e intervenção/aplicação na realidade (BERBELL, 1996).

Com base no estabelecido no Projeto Pedagógico, os discentes desenvolvem atividades integradoras ensino-serviço-comunidade durante todo o curso, sob a orientação de um docente coordenador do eixo a cada período. Os temas a serem trabalhados são definidos de acordo com um tema gerador a partir dos componentes curriculares integrantes de um eixo temático a cada semestre letivo, que direcionam os subtemas. As Atividades Integradoras são socializadas a toda comunidade acadêmica por meio de apresentações e discussões ao final de cada período.

Portanto, a partir do eixo temático "Cuidados de Enfermagem I", do tema gerador "Atuação da enfermagem na saúde" e do subtema "Enfermagem e saúde do 
adolescente" a Atividade Integradora foi desenvolvida segundo as etapas da Metodologia Problematizadora Arco de Charles Maguerez, que visa identificar um problema em dada realidade e, posteriormente, propor soluções e intervir para solucioná-lo ou minimizá-lo.

Diante disso, a escola foi a realidade escolhida, visto que é um ambiente onde o público adolescente é facilmente encontrado. Assim, utilizou-se a seguinte pergunta norteadora: qual problema de saúde, no público adolescente, é mais evidente na realidade escolar e como a enfermagem pode colaborar para mudar esta realidade?

A partir disso, houve o planejamento e execução de cada etapa: observação da realidade, que consiste na participação dos sujeitos/discentes com um olhar atento da realidade e escolher aspectos que precisam ser desenvolvidos, trabalhados, revisados e melhorados, que podemos chamar de "problema". O levantamento dos pontos-chave é o momento de síntese após a escolha do problema a ser estudado e as variáveis determinantes da situação. Na teorização há a busca da compreensão do problema e dos acontecimentos que o desencadeia. A etapa de hipóteses de solução consiste na elaboração de alternativas possíveis para solucionar o problema, de maneira crítica e criativa. E, por fim, a intervenção/aplicação na realidade, é a etapa onde os discentes levam a proposta de solução do problema para a realidade observada, a fim de transformá-la (PRADO et al., 2012).

A figura 1 ilustra o planejamento e execução das etapas da Metodologia Problematizadora Arco de Charles Maguerez, realizada pelos discentes de enfermagem sob orientação docente. 
Figura 1: Planejamento e execução das etapas da Metodologia Problematizadora Arco de Charles Maguerez.

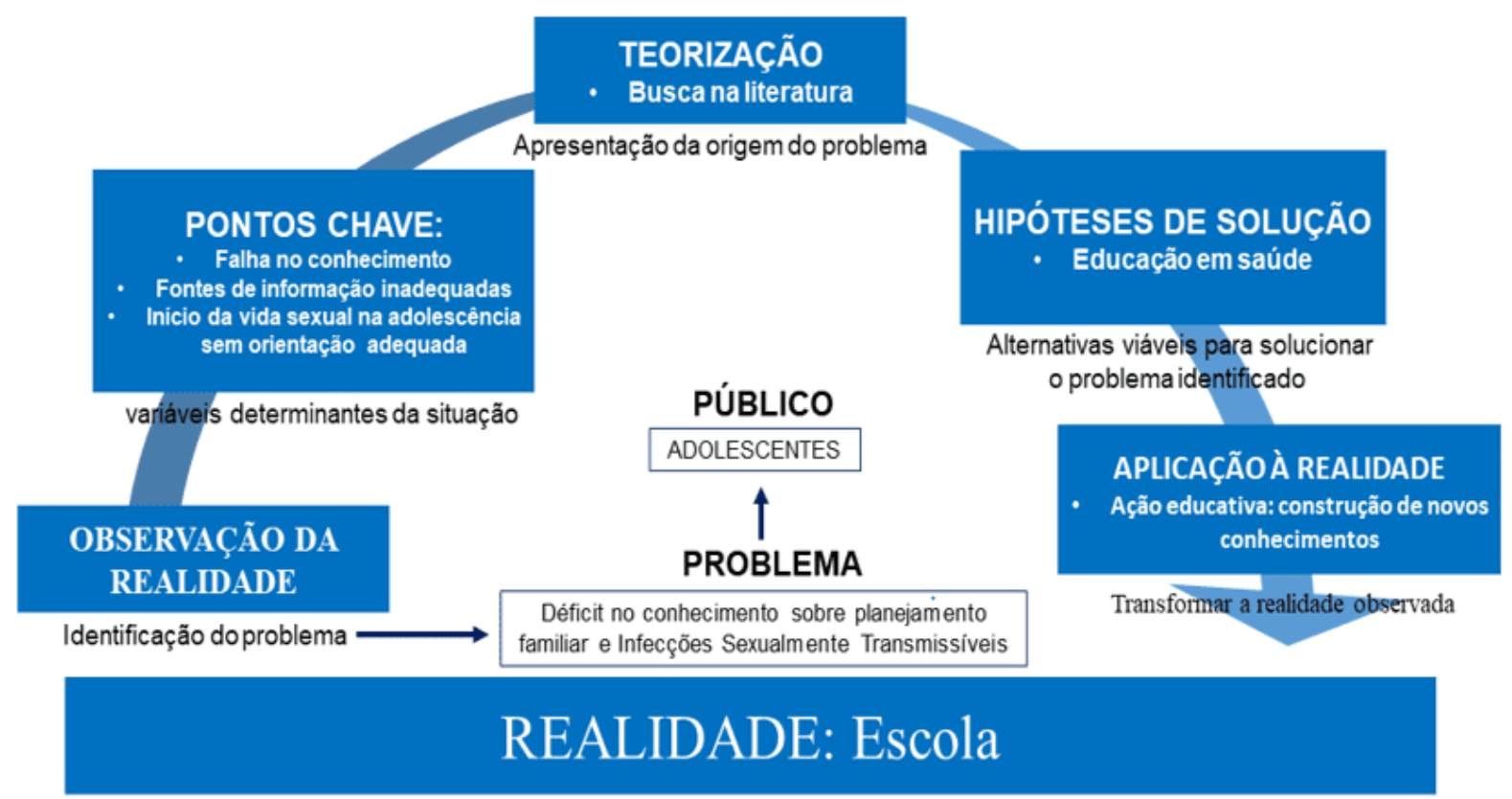

PERGUNTA NORTEADORA: Qual problema de saúde, no público adolescente, é mais evidente na realidade escolar e como a enfermagem pode colaborar para mudar esta realidade?

Fonte: Próprias autoras.

\section{RELATO DA EXPERIÊNCIA}

A princípio, foi realizada a primeira etapa da Metodologia Problematizadora Arco de Charles Maguerez, com a observação da realidade local em busca de uma problemática que a enfermagem pudesse intervir e contribuir positivamente para a saúde do público adolescente. Assim, no dia 25 de março de 2019, foi realizada visita à uma escola pública municipal do sul paraense, na qual compreende as séries do primeiro ao nono ano, onde foi possível fazer a escuta da coordenação e professores da escola.

A falta de conhecimento sobre planejamento familiar e infecções sexualmente transmissíveis foram as principais necessidades observadas, onde, segundo relato dos profissionais presentes, esses adolescentes têm um déficit elevado em relação 
ao assunto, o que já acarretou gravidez indesejada e influência sexual precoce aos demais juvenis.

A escola conta com o apoio da secretaria municipal de educação do município, a qual, uma vez ao ano, realiza palestras voltadas a essa problemática. As experiências com essa metodologia têm se mostrado positiva no ambiente, reduzindo os números de gravidez. No entanto, ainda pode-se observar uma grande lacuna no pensamento crítico desses jovens para o cuidado e prevenção de problemas de saúde na esfera sexual e reprodutiva.

Na segunda etapa do Arco de Charles Maguerez, que corresponde ao levantamento dos pontos chaves, foram levantados possíveis fatores desencadeadores/relacionados ao problema encontrado "déficit no conhecimento sobre planejamento familiar e infecções sexualmente transmissíveis". Assim, temos:

A falha no conhecimento sobre sexualidade e reprodução, como primeiro ponto chave, mencionado por Silva et al. (2016), como algo percebido pelo significativo percentual de desconhecimento em relação à transmissão e prevenção sobre as ISTs/AIDS, o que geram comportamentos de risco e tornam o público adolescente vulnerável.

Um estudo realizado por Oliveira et al. (2009) evidenciou a existência de uma lacuna de informações pelos adolescentes a respeito dos métodos de prevenção das ISTs/AIDS, assim como a existência de crenças que são repassadas e sustentadas pelo seu grupo social.

O segundo ponto chave são as fontes de informação inadequadas, onde a mídia global coopera para a divulgação de conteúdos relacionados à sexualidade, tendo como principal alvo os jovens e adolescentes. Esse fator colabora para o processamento inadequado de informações, visto que não esclarecem ou educam sexualmente estes públicos (PINTO et al., 2013). 
Por fim, o último ponto chave é o início da vida sexual na adolescência sem orientação adequada. A prática sexual na adolescência traz consigo alterações reprodutivas e na saúde do adolescente, que por vezes, assumem condutas sem o conhecimento prévio do assunto, o que pode influenciar no risco de contaminação por ISTs/AIDS e gravidez indesejada (CARNEIRO et al., 2015).

$\mathrm{Na}$ etapa da teorização, buscou-se na literatura embasamento para a fundamentação teórica do problema encontrado. Através disso, foi possível um aprofundamento nos fatores ligados à problemática e métodos de intervenção já utilizados na prática para elencar as hipóteses de solução.

Segundo estimativas da Organização Mundial da Saúde (OMS), mais de um milhão de indivíduos apresentam casos de Infecções Sexualmente Transmissíveis (IST) por dia no mundo inteiro. Com base nisso, vê-se a importância de abordar a educação sexual na adolescência, já que esta implica tanto na saúde do adolescente, quanto em sua vida reprodutiva (FEDERAÇÃO BRASILEIRA DAS ASSOCIAÇÕES DE GINECOLOGIA E OBSTETRÍCIA, 2018).

Através da educação é possível alcançar a reformulação de hábitos e o desenvolvimento intelectual. De acordo com o Código De Ética Dos Profissionais De Enfermagem (2007), Art. 70, o enfermeiro tem como uma de suas responsabilidades promover o ensino entre os membros da sua equipe, bem como para seus clientes.

Partindo desse princípio, a aplicação da Educação em Saúde no ambiente escolar promove a saúde, ensino, aprendizagem e troca de experiências, compactuando-se com a cultura, realidade de vida e conhecimento dos adolescentes. Esse processo pode ser realizado com a utilização de dinâmicas que permitam despertar o senso crítico, autoanálise, ideais sociais, reflexão e exposição de lacunas para que haja uma interação entre o indivíduo/coletivo, propiciando, dessa forma, maior autonomia no autocuidado e em sua vivência (FERNANDES et al., 2019).

Sendo assim, o ambiente escolar propicia aos indivíduos a transformação não somente da sua realidade, mas também a daqueles que o cercam, onde o 
enfermeiro, enquanto educador, atua principalmente no desenvolvimento do autocuidado do adolescente.

Desta forma, a proposta de intervenção fundamentou-se nas ações de educação em saúde que, segundo a Organização Mundial de Saúde (OMS), é um processo baseado em conteúdo científico que utiliza oportunidades educacionais de forma a capacitar os indivíduos, isoladamente ou em conjunto, para tomarem decisões importantes relacionadas à sua saúde (FEIO, OLIVEIRA, 2015).

Assim, realizou-se a última etapa do Arco de Charles Maguerez, que é a intervenção na realidade para minimizar o problema encontrado. À vista disso, desenvolveu-se uma ação de educação em saúde sexual e reprodutiva com os adolescentes, com idade a partir dos 12 anos, regularmente matriculados em uma escola pública municipal de ensino fundamental II, no mês de maio, nos períodos matutino e vespertino.

Para isso, no dia 27 de maio de 2019 foi encaminhado um ofício à escola solicitando autorização para a realização de rodas de conversa sobre sexualidade e gravidez na adolescência. Após o parecer favorável, no dia 4 de junho de 2019, os discentes do $3^{\circ}$ ano do curso de enfermagem da UEP, realizaram as atividades com os alunos da instituição nos períodos matutino e vespertino, com as turmas de $8^{\circ}$ e $9^{\circ}$ ano.

Segundo Silva et al. (2016), ações de educação sexual nas escolas são necessárias para incentivar os adolescentes a terem um comportamento sexual saudável, seguro e menos exposto a riscos.

A abordagem foi feita através de rodas de conversa, na qual foram utilizadas metodologias participativas levando em conta a sensibilidade dos adolescentes e seus conhecimentos já existentes sobre o assunto tratado. Os discentes foram divididos em dois grupos aleatórios para o desenvolvimento das atividades em dois espaços distintos com os adolescentes. 
A princípio, os grupos foram trabalhados com ambos os sexos e, posteriormente, de forma separada. Observou-se maior aproveitamento e participação dos juvenis quando em grupos divididos por sexo, principalmente em relação ao público feminino que, a priori, encontrava-se mais retraído e menos comunicativo. Desta forma, houve melhor direcionamento dos temas de forma a adequar-se às individualidades e necessidades de cada público.

No decorrer das rodas de conversa foi percebido o déficit de conhecimento acerca das ISTS/AIDS e reprodução por parte dos educandos, em vista de não estarem familiarizados com as nomenclaturas das principais Infecções Sexualmente Transmissíveis e a importância do preservativo de barreira na prevenção delas. Alguns dos alunos, durante a realização da dinâmica de "mitos e verdades" relacionados ao tema, relataram ser verdade que o anticoncepcional também previne, além da gravidez, contra ISTs/AIDS.

Além disso, a maioria dos presentes não tinham conhecimento que as ISTs poderiam, também, ser transmitidas durante o sexo anal e oral sem a utilização da camisinha e, que algumas infecções poderiam ser transmitidas sem a penetração dos órgãos genitais, mas sim pelo contato, como é o caso do HPV. Um estudo realizado com estudantes adolescentes acerca do conhecimento do HIV/AIDS mostrou que mais de $90 \%$ dos participantes apresentaram déficit de conhecimento sobre o assunto (ALBUQUERQUE et al., 2012).

Dados semelhantes também foram encontrados em uma amostra com 38 educandos. Os adolescentes foram divididos em dois grupos, onde não houve distinção por sexo, e $87 \%$ de ambos os grupos apresentaram pouco conhecimento sobre o conceito das IST's/AIDS e sua forma de transmissão (CARNEIRO et al., 2015)

Durante a conversa com os adolescentes foi destacado a importância do enfermeiro na orientação sobre sexualidade, principalmente para sanar dúvidas relacionadas às medidas preventivas das ISTs/AIDS e planejamento familiar. Ademais, foi informado que as Estratégias de Saúde da Família oferecem esses recursos preventivos, como 
preservativos masculino e feminino, lubrificante, anticoncepcionais oral e injetável e consultas médica e de enfermagem. Em um estudo realizado por Silva et al. (2020), os adolescentes apresentaram menor conhecimento sobre prevenção da gravidez e aconselhamento e atendimento em saúde sexual e reprodutiva.

No momento, foram apresentados os preservativos masculino e feminino e demonstrado de forma alusiva, com objetos, a maneira correta de abrir a embalagem, como se coloca os preservativos feminino e masculino, sua retirada e descarte correto. Após a demonstração, dois dos adolescentes, do sexo masculino, repetiram o processo, o que contribuiu para reforçar o aprendizado dos educandos. Ainda, foi explicado como armazená-los de forma correta, a importância de utilizá-los íntegros, bem como a não reutilização, a fim de evitar problemas na proteção oferecida por eles.

É válido ressaltar que alguns dos adolescentes relataram que possuem dificuldade ou nunca conversaram sobre assuntos relacionados à sexualidade com os pais. Fonseca, Gomes, Teixeira, (2010) identificaram aprovação dos adolescentes com relação a se trabalhar a temática educação sexual na escola, especialmente por não terem o hábito de conversar sobre o assunto com a família. É imprescindível que a educação sexual se inicie desde a infância, de forma contínua e integrada ao desenvolvimento do infante. Os pais, ou responsáveis, possuem participação importante neste processo de formação, que deve ser complementada pela escola e pelos profissionais da saúde.

Em um estudo realizado por Genz et al. (2017), os autores destacam a importância da adoção de estratégias para promoção da saúde desse público através do fornecimento de informações, citando como exemplo as atividades em escolas que proporcionam interação entre serviços de saúde, professores e família, objetivando assim, garantir a esses adolescentes maior autonomia para práticas sexuais seguras.

Nesse sentido, o profissional de enfermagem, por ter formação generalista, colabora não somente no viés curativo, mas também preventivo, uma interface da sua prática 
que envolve a educação em saúde para o grupo adolescente (OLIVEIRA et al., 2008).

\section{CONSIDERAÇÕES FINAIS}

A falha no conhecimento sobre planejamento familiar e questões ligadas à sexualidade foram problemáticas observadas não só no relato dos profissionais da escola, mas no contato direto com os adolescentes durante a realização da ação educativa. Diante disso, vê-se a necessidade de ações que intensifiquem o cuidado com este público para a promoção da saúde e prevenção de agravos.

O cuidado realizado por meio da educação em saúde é uma estratégia que se mostra eficiente dentro dos espaços onde os adolescentes estão inseridos, especialmente no ambiente escolar. Nesse sentido, a ação desenvolvida colaborou para o conhecimento reflexivo e conscientização que podem propiciar condutas mais responsáveis, cooperando para a prevenção de gravidez na adolescência e ISTs/AIDS.

Ademais, a Metodologia Problematizadora Arco de Charles Maguerez proporcionou aos discentes de enfermagem um olhar mais amplo acerca da saúde sexual e reprodutiva do adolescente, onde o enfermeiro assume um papel de extrema importância como educador, colaborando para o desenvolvimento do autocuidado deste público.

\section{REFERÊNCIAS}

ALBUQUERQUE, Jaqueline Galdino; PINHEIRO, Patricia Neyva de Costa; LOPES, Marcos Venícios Oliveira; MACHADO, Maria de Fátima Antero Sousa. Conhecimento deficiente acerca do HIV/AIDS em estudantes adolescentes: identificação de diagnóstico de enfermagem da NANDA. Revista eletrônica de Enfermagem, Goiânia, v. 14, n. 1, p. 104-111, 2012. Disponível em: https://www.revistas.ufg.br/fen/article/view/12256. Acesso em: 12/06/2019. 
BERBEL, Neusi Aparecida Navas. Metodologia da problematização no Ensino Superior e sua contribuição para o plano da práxis. Semina: v.17, n. esp., p. 7-17, 1996.

CARNEIRO, Rithianne Frota; SILVA, Nalysse Chris da; ALVES, Thais Almeida; ALBUQUERQUE, Danielle de Oliveira; BRITO, Diego Colaço de; OLIVEIRA, Leonice Lima de. Educação sexual na adolescência: uma abordagem no contexto escolar. Sanare, v. 14, $\quad$ n. $01, \quad$ p. 104-108, 2015 . Disponível em: https://sanare.emnuvens.com.br/sanare/article/view/617. Acesso em: 12/06/2019.

CONSELHO FEDERAL DE ENFERMAGEM. Código de ética dos profissionais de enfermagem. Rio de Janeiro, 2007. Disponível em: https://assets.itpac.br/arquivos/Revista/62/6.pdf. Acesso em: 12/06/2019.

FEDERAÇÃO BRASILEIRA DAS ASSOCIAÇÕES DE GINECOLOGIA E OBSTETRÍCIA. Número De Infecções Sexualmente Transmissíveis (IST) aumenta. 2018. Disponível em: ttps://www.febrasgo.org.br/pt/noticias/item/565numero-de-infeccoes-sexualmente-transmissiveis-ist-aumenta. Acesso em: 12/06/2019.

FERNANDES, Joelma Rezende; SILVA, Viviane da Costa Freitas; VERISSIMO, Weslley dos Passos; VIANNA, Nicoly Texeira; CARNEIRO, Mariana Leônidas. Educação em saúde: o papel do enfermeiro como educador em saúde no cenário de IETC. Revista da JOPIC, v. 02, n. 04, p. 2-10, 2019. Disponível em: https://www.unifeso.edu.br/revista/index.php/jopic/article/view/928. Acesso em: 12/06/2019.

FEIO, Ana; OLIVEIRA, Clara Costa. Confluências e divergências conceituais em educação em saúde. Saúde e Sociedade, São Paulo, v. 24, n. 2, p. 703-715, 2015. Disponível em: https://www.scielo.br/j/sausoc/a/gnZXGHdRjfLvGhkY4Jgg6Nz/?format=pdf\&lang=pt. Acesso em 24/09/2021. 
FONSECA, Adriana Dora da; GOMES, Vera Lúcia de oliveira; TEIXEIRA, Karina Correa. Percepção de adolescentes sobre uma ação educativa em orientação sexual realizada por acadêmicos(as) de enfermagem. Revista de Enfermagem Escola Anna Nery, Rio de Janeiro, v. 14, n. 2, p. 3030-337, 2010. Disponível em: http://bases.bireme.br/cgi-

bin/wxislind.exe/iah/online/?IsisScript=iah/iah.xis\&src=google\&base=LILACS\&lang=p \&nextAction=Ink\&exprSearch=553806\&indexSearch=ID. Acesso em: 10/06/2019.

GENZ, Niviane; MEINCKE, Sonia Maria Könzgen; CARRET, Maria Laura Vidal; CORRÊA, Ana Cândida Lopes; ALVES, Camila Neumaier. Doenças sexualmente transmissíveis: conhecimento e comportamento sexual de adolescentes. Texto Contexto Enfermagem, v. 26, n. 2, e5100015, 2017. Disponível em: https://www.scielo.br/j/tce/a/GrXZhcDdPFwm5Q8TMSZbxMt/?lang=pt. Acesso em: 18/06/2019.

INSTITUTO BRASILEIRO DE GEOGRAFIA E ESTATÍSTICA. Estatísticas de Gênero - Indicadores sociais das mulheres no Brasil. Brasília, 2021. Disponível em: https://www.ibge.gov.br/estatisticas/multidominio/genero/20163-estatisticas-degenero-indicadores-sociais-das-mulheres-nobrasil.html?edicao=20164\&t=downloads. Acesso em: 29/09/2021.

LIMA, Iris Angélica; MOREIRA, Maria Sebastiana da Silva. Importância do enfermeiro na escola de ensino fundamental: revisão da literatura. 2018. $19 \mathrm{f}$. Trabalho de Conclusão de Curso (bacharelado em enfermagem) - Centro Universitário São Lucas, Porto Velho, 2018. Disponível em: http://repositorio.saolucas.edu.br:8080/xmlui/bitstream/handle/123456789/2765/Lima \%2C\%20lris\%20Ang\%C3\%A9lica\%20de\%2CMoreira\%2C\%20Maria\%20Sebastiana \%20da\%20Silva\%20-

\%20Import\%C3\%A2ncia\%20do\%20enfermeiro\%20na\%20escola\%20de\%20ensino \%20fundamental\%20revis\%C3\%A30\%20de\%20literatura.pdf?sequence=1\&isAllowe $\mathrm{d}=\mathrm{y}$. Acesso em: 18/06/2019. 
OLIVEIRA, Denize Cristina de; PONTES, Ana Paula Munhen de; GOMES, Antônio Marcos Tosoli; RIBEIRO, Monique Carvalho Marrafa. Conhecimentos e práticas de adolescentes acerca das DST/HIV/AIDS em duas escolas públicas municipais do Rio de Janeiro. Revista de Enfermagem Escola Anna Nery, Rio de Janeiro, v. 13, n. $4, \quad$ p. 833-841, 2009. Disponível em: https://www.scielo.br/j/ean/a/TmChg6gRBX4N9jHwbkyZSBc/?format=html\&lang=pt. Acesso em: 12/06/2019.

OLIVEIRA, Thays Cristina de; CARVALHO, Liliane Pinto; SILVA, Marysia Alves da. O enfermeiro na atenção à saúde sexual e reprodutiva dos adolescentes. Revista Brasileira de Enfermagem, Brasília, v. 61, n. 3, p. 306-311, 2008. Disponível em: https://www.scielo.br/j/reben/a/r8KTg7hZmqX9NwVhT3hJCQs/abstract/?lang=pt. Acesso em: 12/06/2019.

PINTO, Maria Benegelania; SANTOS, Nathanielly Cristina C. B.; ALBUQUERQUE, Adriana Montenegro de; RAMALHO, Marclineide Nóbrega de Andrade; TORQUATO, Isolda Maria Barros. Educação em saúde para adolescentes de uma escola municipal: a sexualidade em questão. Ciência, Cuidado e Saúde, v. 12, n. 3, p. 587-592, 2013. Disponível em: https://periodicos.uem.br/ojs/index.php/CiencCuidSaude/article/view/18470. Acesso em: $12 / 06 / 2019$.

PRADO, Marta Lenise; VELHO, Manuela Beatriz; ESPÍNDOLA, Daniela Simoni; SOBRINHO, Sandra Hilda; BACKES, Vânia Marli Schuber. Arco de Charles Maguerez: refletindo estratégias de metodologia ativa na formação de profissionais de saúde. Escola Anna Nery, Rio de Janeiro, v. 16, n. 1, p. 172-177, 2012 Disponível em: https://www.scielo.br/j/ean/a/89NXfW4dC7vWdXwdKffmf4N/?format=pdf\&lang=pt. A cesso em: 12/06/2019.

SILVA, Richardson Augusto Rosendo da; NELSON, Ana Raquel Cortês; DUARTE, Fernando Hiago da Silva; PRADO, Nanete Caroline da Costa; COSTA, Danyella Augusto Rosendo da Silva; HOLANDA, Jose Rebberty Rodrigo. Conhecimento de 
estudantes adolescentes sobre transmissão, prevenção e comportamentos de risco em relação às DST/HIV/AIDS. Revista de Pesquisa: Cuidado é Fundamental Online, v. 8, n. 4, p. 5054-5061, 2016. Universidade Federal do Estado do Rio de Janeiro UNIRIO. Disponível em: http://www.seer.unirio.br/index.php/cuidadofundamental/article/view/3634/pdf_1. Acesso em: 12/06/2019.

SILVA, Sílvia Manuela Dias Tavares da; FERREIRA, Maria Margarida da Silva Vieira; AMARAL-BASTOS, Maria Manuela; MONTEIRO, Maria Amélia José; COUTO, Germano Rodrigues. Diagnóstico do conhecimento dos adolescentes sobre sexualidade. Acta Paulista de Enfermagem, n. 33, p. 1-7, 2020. Disponível em: https://www.scielo.br/j/ape/a/DWD9fVf3Nj6Dx3GVGSCDYrd/?lang=pt\&format=pdf. Acesso em 12/06/2019.

UNIVERSIDADE DO ESTADO DO PARÁ (UEPA). Projeto Pedagógico do Curso de Graduação em Enfermagem. Escola de Enfermagem Magalhães Barata- EEMB. Belém: Universidade do Estado do Pará, 2013.

Enviado: Setembro, 2021.

Aprovado: Dezembro, 2021. 